\title{
Design Recommendations for Augmenting Creative Tasks with Computational Priming
}

\author{
Jonas Oppenlaender \\ University of Oulu \\ Oulu, Finland \\ jonas.oppenlaender@oulu.fi
}

\author{
Simo Hosio \\ University of Oulu \\ Oulu, Finland \\ simo.hosio@oulu.fi
}

\begin{abstract}
Supporting creativity is a grand challenge in HCI. A critical component of creativity is the ability for divergent thinking, and divergent thinking can be fostered through looking at the problem through the lens of a different person, by assuming a role. Prior work found that assuming a role and affective stimulation with images may lead individuals to be more creative. In this work, we investigate the use of roles in stimulating the creativity of individuals in two complementary studies. In the first study, we implemented an online instrument for augmenting creativity with roles and images, and recruited crowd workers $(n=60)$ to complete a divergent thinking task while assuming a role. Interestingly, and in contrast to earlier findings, our analysis could not confirm the computational priming having an effect on the outcome of a small batch of creative tasks. In the second study, we observed the effect of roles on the ideation process of individuals when they reach an impasse in the flow of ideas. Our complementary studies highlight that adopting roles can help when one runs out of ideas, but this is not a silver bullet for improving divergent thinking, especially in online crowdsourcing environments that are increasingly being used for experiments and data collection in behavioural science. Our work informs the design of future crowd-powered creativity support tools and contributes a timely case study to the body of literature in the growing field of creativity support online.
\end{abstract}

\footnotetext{
Permission to make digital or hard copies of all or part of this work for personal or classroom use is granted without fee provided that copies are not made or distributed for profit or commercial advantage and that copies bear this notice and the full citation on the first page. Copyrights for components of this work owned by others than the author(s) must be honored. Abstracting with credit is permitted. To copy otherwise, or republish, to post on servers or to redistribute to lists, requires prior specific permission and/or a fee. Request permissions from permissions@acm.org. MUM 2019, November 26-29, 2019, Pisa, Italy

(c) 2019 Copyright held by the owner/author(s). Publication rights licensed to ACM.

ACM ISBN 978-1-4503-7624-2/19/11 ..\$15.00

https://doi.org/10.1145/3365610.3365621
}

\section{CCS CONCEPTS}

- Human-centered computing $\rightarrow$ Human computer interaction (HCI); User studies; • Information systems $\rightarrow$ Crowdsourcing.

\section{KEYWORDS}

Crowdsourcing, creativity support, computational priming, creativity

\begin{abstract}
ACM Reference Format:
Jonas Oppenlaender and Simo Hosio. 2019. Design Recommendations for Augmenting Creative Tasks with Computational Priming. In MUM 2019: 18th International Conference on Mobile and Ubiquitous Multimedia (MUM 2019), November 26-29, 2019, Pisa, Italy. ACM, New York, NY, USA, 13 pages. https://doi.org/10.1145/3365610. 3365621
\end{abstract}

\section{INTRODUCTION}

Paid online labour markets have rapidly developed into a way to cost-effectively tap into the collective insights of diverse crowds [22, 27, 35]. Crowds consist of individuals, and an individual's creativity can be enhanced through creativity support tools $[56,57]$. A key research area concerning these tools is the ideation phase [25, 66]. Prior work suggests that computational priming in the ideation phase (e.g. by asking individuals to assume roles or by showing an affect-laden image) may lead to ideas that are more creative [40, 46, 63]. Lewis et al., for instance, found small but significant evidence that "positive affect-laden images positively influence idea generation" [40].

In this paper, we investigate augmenting an individual's thinking process through adopting different roles in two complementary studies. In the first study, we investigated the effect of impersonating a self-selected role on the creativity of individual crowd workers when working on a batch of simple divergent thinking tasks. Workers $(n=60)$ underwent three counterbalanced conditions sequentially: a control condition (generating ideas without a role), and two priming conditions (generating ideas while impersonating a role and while impersonating a role depicted in a stimulating image). We evaluated the creativity of the ideas and quantitatively investigated how workers perceive the roles as a tool to accomplish the task. 
In the second study, we evaluated strategies of using roles in the ideation phase when faced with an impasse in the flow of ideas. These strategies include: self-selecting multiple roles to solve the task, self-selecting only one role, and being assigned a role.

Interestingly, the results of the first study are not in line with the findings of related research [40, 46, 63]. We found that when adopting a role and working on small batch of standard creative tasks, online crowd workers are not more likely to generate ideas that are more creative. The results of the second study, however, highlight that - if given at the right time and for the right problem - roles constitute an appropriate tool for overcoming an impasse in the flow of ideas and stimulating creativity. Our findings suggest that successfully using roles to encourage divergent thinking must involve planning and consideration for how the roles are framed, when the roles are used, and whether the roles are familiar to participants, without introducing too many choices in the task which can slow people down and affect their task performance and their perception of task difficulty.

Our complementary studies contribute an incremental and unexpected result to the science of creativity and its application in the context of crowdsourcing: adopting roles is not a silver bullet for improving creativity in online crowdsourced environments. Our contradictory findings help crowdsourcing researchers and practitioners begin to understand some of the benefits and limitations of the use of roles in stimulating the crowd's creativity and supporting divergent thinking.

\section{RELATED WORK}

Crowdsourcing [33] - as a contemporary means of reaching vast crowds online for completing work distributed in small chunks - offers unprecedented opportunities for outsourcing creative work [64]. Humans excel in recombination, analogical transfer and divergent thinking while machines fall short in these fundamental characteristics needed for creativity [34]. Our work is situated within the intersection of three key areas that unlock the potential of individuals by augmenting creativity.

\section{Wisdom of Many from the Single Individual}

To more efficiently elicit information, researchers have investigated whether the mechanism of wisdom of the crowd [62] can be applied to a single individual. In these experiments, information was sourced not from the crowd, but from a single individual and measures were employed to increase the diversity of the individual's responses.

Herzog and Hertwig presented dialectical bootstrapping as an approach to improve estimates from a single person [31]. The approach aims at reducing a person's error by averaging two guesses made under different conditions. The second guess is geared towards making the person draw from an at least partly different set of knowledge than the first guess. While the individual may assume the first guess is reliably reflecting the best information available, a forced second guess may contribute additional information [65]. The average of the two guesses is expected to bracket the ground truth $[36,37,42]$, thereby simulating the wisdom of many.

The above method is geared towards improving quantitative estimates of a known fact. In studies involving divergent thinking, however, answers are highly subjective and no ground truth is available [35]. The approach described above nevertheless demonstrates that a person's creative performance can be increased using different stimuli. One key enabling factor of this augmentation in a person's creative ability is the manipulation of context.

\section{Computational Priming in Crowdsourcing Tasks}

In most things that humans do, context matters. Similarly, the task environment - i.e., context - of a crowdsourcing task may affect the outcome of the task [17]. Context, in this case, refers to any information that can be used to characterise the situation of the crowd worker [23]. Visual computational priming manipulates this context and activates behaviour via exposure to a stimulus [46].

The manipulation of context has proven to be a fruitful ground for recent research. For instance, IT-enabled stimuli may help individuals explore their knowledge base more deeply [3]. Cognitive priming has been used to improve electronic team brainstorming sessions [21]. August et al. showed that contextual framing affects the motivation of participants for taking part in a study [8]. Chandler and Kapelner came to the same conclusion, and further showed that framing a crowdsourcing task may lead to an increase in the quantity and quality of the task outcome [13]. De Rooij et al. investigated priming with avatars and creative stereotypes [20, 43]. Their results suggest that a non-creative avatar may diminish creativity, but a creative avatar may not augment creativity.

Nguyen et al. showed that framing a task with positive affective language may reduce the frustration of participants and lead to an increase in work quality [48]. The framing of a task may also influence the attention of a worker [9]. Morris et al. applied affective priming theory in crowdsourcing [46]. The authors found weak, but significant evidence that priming with positive stimuli in the form of images in the ideation phase increases the originality of the ideas created. Lewis et al. applied affective computational priming to Guilford's Alternative Uses task [28], a standard test of divergent thinking, by giving study subjects negative, neutral or positive stimuli in the form of an image [40]. Based on their findings, the authors suggest that priming with positive affect-laden images positively influences the quality of generated ideas. 


\section{Analogical Thinking to Support Creativity}

Analogical thinking is another method of augmenting one's creativity. Information from unrelated and distant domains has been shown to be inspirational and applying foreign skills, methods and tools from another domain may lead to creative solutions [71]. Engineers, for instance, often take inspiration from nature in solving problems. Similar strategies can be observed in everyday ideation where individuals use objects and information they discover as creative resources for developing and shaping ideas [41].

Analogical thinking is an example of the human ability to transcend the limits of reasoning within their own domain of expertise. Humans "can also reason about beliefs which would arise under hypothetical scenarios" [53]. This human ability spurred research into supporting creativity by making individuals assume roles. Teevan and $\mathrm{Yu}$ investigated whether assuming roles while solving a creative task inspired people to produce ideas that are more creative [63]. In their study, the mechanism of the "wisdom-of-an-individualassuming-roles" spurred the participants to come up with ideas of higher perceived creativity than without roles.

As a summary, there exist only a few case studies that specifically look into augmenting the creativity of workers in online labour markets. Further, while the evidence in the studies suggests that roles may indeed help boost the creativity of individuals, further research is more than warranted, given that these marketplaces are becoming increasingly important research assets for behavioural science and for industry use cases [52].

\section{STUDY 1: AUGMENTING THE CROWD WITH ROLES}

Our first study aims to augment the creativity of individual workers online. We build on prior work by Teevan and $\mathrm{Yu}$ [63], Lewis et al. [40], and Morris et al. [46]. In a withinsubject study, we explore the effect of priming with a role on the creativity of individual crowd workers while they work on a small batch of divergent thinking tasks. We formulate the following hypotheses.

H1. Participants with a positive attitude towards the roles will produce ideas that are more creative than participants with a negative attitude.

Based on prior work [40, 46, 63, 70], we expect that roles allow crowd workers to increase their divergent thinking abilities and produce ideas of greater creativity. We expect that, overall, participants will have a positive attitude towards adopting roles as helpful cognitive tools in accomplishing the task. We further expect that online workers who have a positive attitude towards the roles will produce ideas that are more creative than workers who do not care as much.
H2a. Priming with a role reduces the time taken to come up with ideas.

H2b. Priming with a role and an additional image reduces the time taken to come up with ideas even further.

Some scholars have advocated using time as a measurement of creativity support [32]. Other scholars have criticised the use of time. For instance, a long time spent on a task may be a sign of a high level of creative engagement, but may not be indicative of the creativity of a person [11]. While we acknowledge that time spent on a task may not be a direct measure for a person's creativity, we still hypothesise that when supported by assuming a different role, a person has to expend less effort to come up with ideas than without a role, thus being able to come up with ideas more quickly.

\section{Study Design Consideration}

To provide a comparison point for our results, we decided on Guilford's Alternative Uses task [28] that has been used in many prior studies, e.g. $[40,46]$. In the task, participants generate as many uses for a common object (e.g. a brick or a paperclip) as possible until they run out of ideas. The number of ideas is used as a measure of creativity in this test.

In our study, we modified the task and limited the number of ideas per condition for three reasons. First, as suggested by Snyder et al., fluency - i.e., the number of ideas - does not necessarily equate to originality and creativity of the ideas [61]. Second, extrinsically motivated crowd workers are unlikely to spend a lot of time and thought on simple, repetitive tasks [26]. Therefore, it is difficult to assess whether a worker genuinely ran out of ideas in one of our study's conditions or simply wanted to move on after contributing what seems like a passing amount of ideas. Our setup tries to mitigate this "spill-over" effect from one condition to another where workers continue to generate ideas, irregardless of the priming condition. Finally, the commonly used way for scoring creative thinking tasks - the combination of frequency (number) and uniqueness (rarity) of ideas - has been criticised for leading to confounding results $[59,68]$. This is because more answers will inevitably lead to a higher probability of there being unique answers [59, 60]. Assessing the uniqueness is the most common way of scoring creative tasks that require divergent thinking [59]. For these reasons, we settled on evaluating the ideas from one perspective only: the unusualness of the generated ideas.

We designed a repeated measures study with three counterbalanced conditions to minimise the effect of a single individual's personal characteristics on the task outcome. Each crowd worker was given one task and completed three conditions sequentially. We describe the procedure in the following section. 

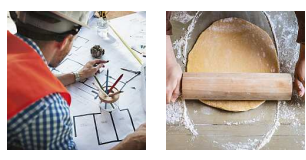

Baker

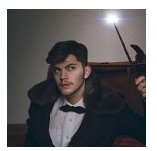

Magician

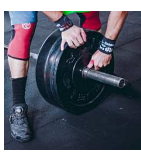

Bodybuilder

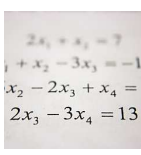

Mathematician

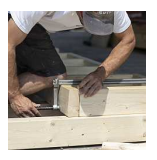

Carpenter

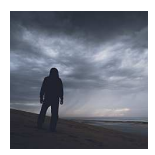

Meteorologist

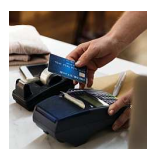

Cashier

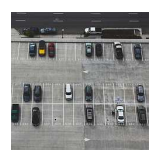

Parking Lot Attendant

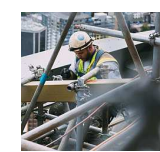

Construction

Worker

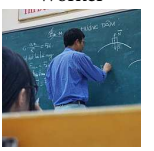

Physicist
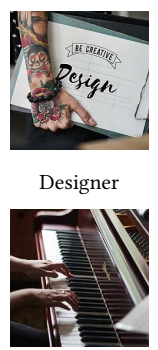

Pianist

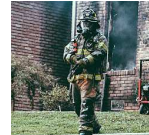

Firefighter

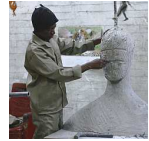

Sculptor

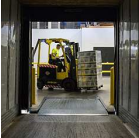

Forklift

Operator



Topologist

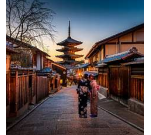

Expert on Japa-

nese Aesthetics Landscaper

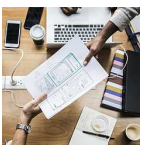

User Interface Designer
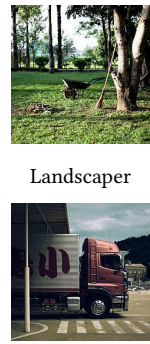

Warehouse Dock Loader

Figure 1: The list of roles and images used for priming.

\section{Procedure}

We invited participants from Prolific ${ }^{1}$ - an online labour market designed for behavioural and user research - to assume self-selected roles while providing answers to the Alternative Uses task. The online workers were asked to provide ideas for unique and unusual uses of a common object. Half the participants were assigned a brick as object, the other half a paperclip. We developed a custom web-based survey instrument to administer the study. The survey instrument and procedure were carefully piloted with three members of Prolific before launching the actual study.

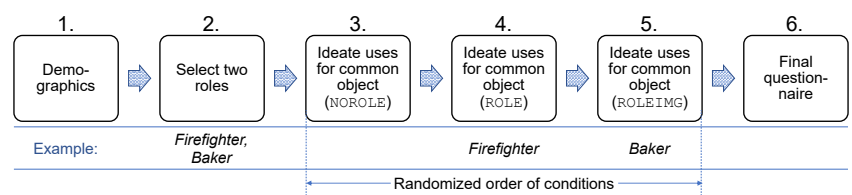

Figure 2: Overview of the stages of Study 1.

Survey Stages. Figure 2 summarises the six distinct stages of the study. Each stage was presented to the participant on a separate web page. After providing basic demographic information in the first stage, participants were instructed to select two different and familiar roles from a list of roles. The 22 roles (see Figure 1) were adapted from Yu et al. [71] who originally elicited the roles from crowd workers in response to two design problems.

In each of survey stages 3-5 (see screenshots in Figures 3a$3 c$ ), workers were instructed to come up with four unique and unusual uses for the respective object (brick or paperclip) under different conditions.

Priming Conditions and Task Instructions. Participants sequentially completed three different conditions (see Table 1). The order of the conditions was randomised and fully counterbalanced. In the NOROLE condition (Figure 3a), we adopted the instructions from related literature [40, 46, 63]. In the

\footnotetext{
$\overline{1_{\text {www.prolific.ac }}}$
}

Table 1: Task instructions in each condition.

\begin{tabular}{ll} 
Condition & Instruction \\
\hline NOROLE & "In this task, your goal is to think of unique and un- \\
& usual uses for a $<$ PAPERCLIP/BRICK>. For example, \\
& using a $<$ paperclip/brick $>$ as an earring is an unusual \\
& and unique use. However, using a $<$ paperclip to bind \\
& papers/brick to build a wall $>$ is not unique or unusual." \\
ROLE & "Imagine you are a $<$ ROLE $>$. As a $a$ ROLE $>$, think of \\
& unique and unusual uses for..." \\
ROLEIMG & "Imagine you are a $<R O L E>$. $<I M A G E>$. As a $<$ ROLE $>$, \\
& think of unique and unusual uses for..."
\end{tabular}

ROLE condition, the instructions were slightly modified (see Table 1 and Figure 3b). The ROLEIMG condition further extended the instructions by additionally priming participants with an image depicting the role (Figure 3c). The images used for priming were taken from Unsplash, a stock photography website, by searching for the name of the role and, in some cases, iteratively expanding the search until an appropriate image was found. The full set of images, as well as the source code of the custom-coded survey instrument, are available in a repository on GitHub ${ }^{2}$.

The specific instructions on each stage were followed by a set of explanations:

This task is spread over 3 stages. Stage $<1 / 2 / 3>$ is below. Your answers must, however, be unique across all stages. Please provide 4 different answers - one answer per textbox below. There is no minimum or maximum word count, simply explain the use case concisely.

DO NOT (!) use any external sources (e.g., websites, people) to complete this task.

In the final stage, we inquired about the overall difficulty of the task, as well as the self-rated novelty and usefulness of the created uses for the object. We also were interested in investigating how crowd workers perceive the roles. To

\footnotetext{
${ }^{2}$ https://github.com/joetm/crowd-roles
} 


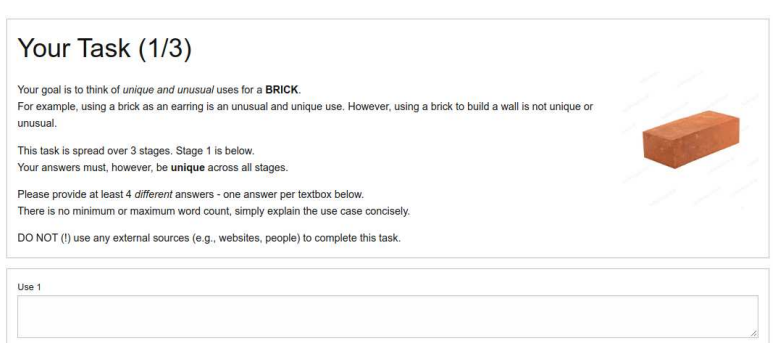

(a) NOROLE condition

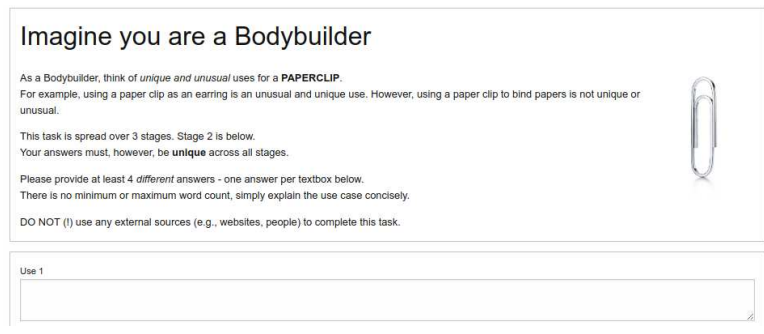

(b) ROLE condition

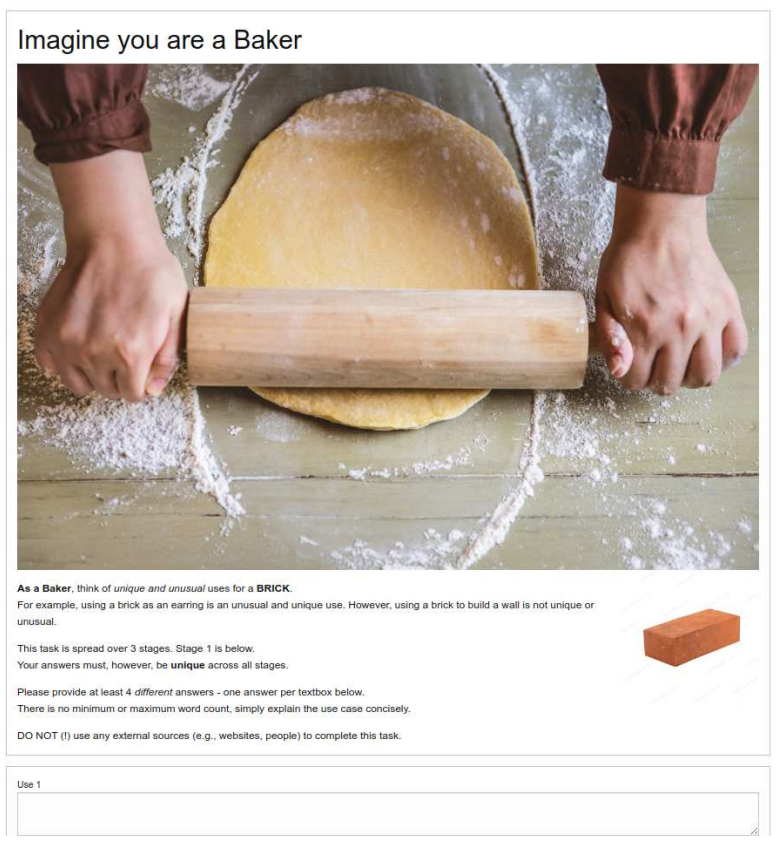

(c) ROLEIMG condition

Figure 3: Screenshots of the three task conditions. Figures 3a and $3 c$ depict the brick task, Figure $3 b$ the paperclip task.

this end, we adapted psychometric questionnaire items from the literature to measure the attitude towards the roles [2], the perceived usefulness of the roles in accomplishing the task [19], and the engagement of the participant, as measured by a super-ordinate construct composed of interest, enjoyment, and concentration $[29,55]$.

\section{Participants}

We invited native-English-speaking members from Prolific in small batches $(n=4)$. To maximise the diversity in our sample, the intake was opened at different days of the week and at different times of the day over a one month period. The study took $15.37 \mathrm{~min}$ on average to complete $(S D=9.93 \mathrm{~min})$. Each participant was paid UK $£ 2.25$ resulting in an average hourly pay of $£ 8.78$ (UK minimum wage at the time: $£ 7.83$ ). Prior to completing the study, all participants were asked for their consent and had the option to cancel their participation at any time.

Our only screening criteria was English as first language. We controlled for cheating behaviour with two questions. Participants were reminded to answer the questions truthfully and that the answer would not affect their compensation. We identified 16 participants who admitted to having used external information sources to complete the task and eleven participants who had completed a similar study earlier. We fully compensated these participants, but excluded their answers from further analysis. We further excluded two participants who did not enter unique uses for the common object. We continued running the study until each of the 12 treatment combinations in our counterbalanced design was filled with five participants.

Participant Demographics. The 60 participants in our final sample (33 male, 24 female, three of unreported gender) were aged from 18 to $64(M=34, S D=11)$ years. The participants had completed on average 173 studies on Prolific $(S D=207)$ with a low number of rejections $(M=0.78, S D=1.27)$. Half the participants were in full-time employment, and six worked part-time. Ten participants were not in paid work (e.g. homemaker, retired or disabled), eight unemployed (and job seeking), and six did not report their employment status. The sample contained eight students.

\section{Results}

In this section, we first present an evaluation of the created ideas. We then summarise the questionnaire responses before we analyse the results in regard to the research questions. We report the results on a 7-point Likert scale from 1 (Strongly Disagree) to 7 (Strongly Agree), if not otherwise mentioned.

Evaluation of the Ideas. A brief analysis of the ideas suggests that the ideas were vastly differing in their representation (see the examples in Table 2). The roles seemed to have helped workers to come up with ideas that were more nuanced (detailed or geared toward a specific use case) than without the role. Further, an analysis of the number of words per idea revealed that the ROLEIMG condition produced, on average, longer ideas. A pairwise t-test confirmed the difference as significant for the paperclip task (see the bottom of Table 3). 
Table 2: Examples of the brick task by P59 (longest time taken overall and in each condition) and P41 (shortest time overall).

\begin{tabular}{llll} 
Worker & NOROLE & ROLE & ROLEIMG \\
\hline P59 & Place [a brick] next to you when sitting & (Mathematician) Use the sides of the & (Physicist) Placing [bricks] on the soles of \\
on a bus to reserve that spot for your & brick to derive mathematical equations & your feet to insulate yourself from elec- \\
friend who will be getting on at a later & that represent one example of random & tric shocks in a thunderstorm. \\
stop. & $\begin{array}{l}\text { impurities on a flat surface. } \\
\text { (Cashier) As a weapon }\end{array}$ & (Baker) To flatten dough
\end{tabular}

To further gauge the creativity of the ideas, we hired workers from Amazon Mechanical Turk (MTurk). The workers were asked to identify the most unusual use for the common object from a randomly-sampled triplet consisting of one participant-provided use per condition (see Figure 4). The meaning of "unusualness" was explained in the task instructions with the same examples used in the main study. Workers were blind to the conditions and paid US $\$ 0.03$ per evaluated triplet. In total, we evaluated 120 randomlysampled triplets - 60 for the paperclip and 60 for the brick. Each triplet was assigned to three crowd workers. The data was aggregated with a majority voting rule, i.e., we counted one vote for a condition if at least two workers agreed on the condition.

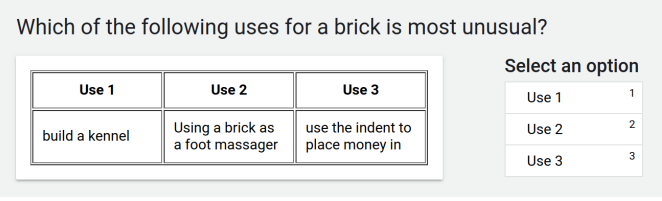

Figure 4: Example of the external evaluation task on MTurk.

The MTurk workers fully agreed in about one third (30.8\%) of the triplets. In $59.2 \%$ of the cases, the third vote was used as a tie breaker to form an agreement on one of the three conditions. In the rest of the cases (10\%), no agreement between the three raters was achieved. We report these cases separately and do not consider them in our evaluation.

Given this evaluation of ideas in triplets, no single condition could be determined to produce the most unusual uses for the respective object (see the top of Table 3). For the brick, the choices of the crowd workers were evenly distributed over the three conditions. For the paperclip task, the MTurk workers voiced a slight preference for the uses produced when being primed with a role and an additional image.

Perceived Difficulty of the Task and Self-rated Performance. On average, the participants thought the task was moderately difficult $(M=2.15, S D=0.92$; on a scale from 1 (Very Difficult) to 5 (Very Easy)). Participants were moderately challenged by the task $(M=5.53, S D=1.10)$, and did not think of themselves as being particularly skilled $(M=3.35$, $S D=1.29)$ or very good at the task $(M=4.20, S D=1.55)$.
Table 3: Top: Count of most unusual ideas per condition in the external evaluation. Bottom: Average number of words per idea and condition.

\begin{tabular}{llcccc} 
& Task & NOROLE & ROLE & ROLEIMG & $\begin{array}{c}\text { Disagree- } \\
\text { ment }\end{array}$ \\
\hline \multirow{2}{*}{ Idea count } & Brick & 18 & 17 & 18 & 7 \\
& Paperclip & 16 & 17 & 22 & 5 \\
\hline \multirow{2}{*}{ Idea length } & Brick & 9.53 & $10.16^{\dagger}$ & $10.89^{\dagger}$ & \\
& Paperclip & 8.15 & $9.85^{*}$ & $10.31^{*}$ & \\
\hline & & & & $\dagger(\mathrm{p}>.05), *(\mathrm{p}<.05)$
\end{tabular}

The task was nevertheless perceived as engaging $(M=4.76$, $S D=.69)$. Participants reported a high level of concentration $(M=6, S D=.90)$. The task was enjoyable $(M=4.80, S D=$ $1.52)$, but only slightly interesting $(M=4.11, S D=.63)$.

On average, the participants rated their set of created ideas as average in both novelty $(M=4.08, S D=1.20)$ and usefulness $(M=3.80, S D=1.47)$, as measured on a Likert-scale from 1 (Not Novel/Not Useful At All) to 7 (Very Novel/Very Useful).

Perception of the Roles in Solving the Task. Interestingly, the participants did not perceive the roles as making the task easier to complete $(M=3.68, S D=1.63)$ nor did they think the roles made them more effective in completing the task $(M=3.93, S D=1.66)$. The participants also thought the roles did not help in accomplishing the task more quickly $(M=3.67, S D=1.94)$. Overall, the participants regarded using roles as only a little useful in accomplishing the Alternate Uses task $(M=3.8, S D=1.86)$. Similarly, the attitude of the participants towards roles was, on average, only slightly positive $(M=4.15, S D=1.68)$.

In summary, while participants had, on average, a slightly positive attitude towards the roles, participants seemed to be not entirely decided on whether the roles helped them in completing the task. This was also reflected in some of the qualitative feedback received from participants: "I' $m$ not sure if the roles helped or hindered my ability to come up with uses for the brick" (P51).

Influence of Attitude towards the Roles on the Creativity of Ideas (H1). To analyse whether the attitude towards adopting roles has an effect on the creativity of the ideas, we grouped 
the participants into two groups: participants with a negative attitude towards the roles (Likert ratings $1-3 ; 17$ participants) and with a positive attitude (ratings $5-7 ; 22$ participants).

Participants with a negative attitude towards the roles thought the task was more difficult $(M=1.76, S D=.66)$ than participants with a positive attitude $(M=2.32, S D=$ $.95), t(36.73)=2.15, p<.05, d=.66$. Both groups rated the novelty of their ideas in a similar way $(M=4, S D=$ 1.41 versus $M=4.14, S D=1.21), t(31.48)=.32, p>.05$. Participants with a positive attitude rated the usefulness of their own ideas slightly higher than participants with negative attitude $(M=4.27, S D=1.39)$ and $(M=3.88$, $S D=1.58), t(32.10)=.81, p>.05$.

Influence of Roles on Task Completion Times (H2). The three conditions took between $2.5 \mathrm{~min}$ (2 users) and $50 \mathrm{~min}$ (P59) to complete. Intuitively, we expected the participants to come up with ideas more quickly when given a role $(\mathrm{H} 2 a)$ and when stimulated with an image depicting the role $(H 2 b)$. A Friedman test did not, however, reveal significant differences between the time taken for the conditions (means: $210.9,223.2,259.0$ seconds for conditions NOROLE, ROLE, and ROLEIMG, respectively), $\chi^{2}=4.43, d f=2, p>0.05$.

Inspecting the data, we found that the ROLEIMG condition had more outliers than the other two conditions. The means, and in particular one of the outliers (P59), showed a clear progression, but inverse to what we initially predicted: P59 spent most time on the condition with the image and the least time on the condition without a role. The majority of the participants seem to however have been unaffected by the given roles when working on the small batch of tasks.

\section{STUDY 2: SUPPORTING CREATIVITY WITH ROLES}

To investigate the effect and impact of assigning roles on creativity further - and to shed light on what might have caused the results in the online study - we organised dyadic interviews with eight students from our campus. Dyadic interviews are a useful approach when analysing a shared experience [45]. We decided on this approach, as individual differences within the interviewed groups were less important and instead, we wanted to draw from the synergy between both interview partners and shed light on additional information that may not have been discussed had we interviewed participants individually. Holding joint interviews also had a pragmatic reason. A brainstorming session can potentially last a very long time. By having the participants announce that they finished the brainstorming, we created a social dynamic that prevented participants from dwelling too long (and potentially unproductively) on the task. The interviews lasted between 10 and 25 minutes. We compensated each student with a coffee voucher worth US $\$ 5$.

\section{Procedure}

The participants (P1-P8; 6 men, 2 women, aged 19 to 29 years; six Bachelor, one Master and one PhD student) were invited in groups of two. The two participants in each group were first briefed about the study and its procedure and then asked to select between a brick or paperclip as object. The participants then completed the Alternative Uses test individually. Participants were instructed to write down unique, useful, but unusual uses for the object (brick or paperclip) of their choice. The meaning of "unusualness" was explained with the same examples used in the online study. The participants were instructed to signal when they started struggling with coming up with new ideas.

Once both participants had depleted their initial repertoire of ideas, the participants were asked to complete the same task with the same object, but under a different condition. Participants in the first group (P1-P2) were allowed to freely consider several roles from a list of possible roles (baker, carpenter, construction worker, user interface designer, barber, nanny, and firefighter). Participants in the second and third group (P3-P6) were instructed to choose exactly one role. The latter better mimics the online study, but we were also curious about what would happen if people were allowed to freely consider several roles at a time and switch between them, if necessary. Lastly, participants in the last group (P7-P8) were assigned a random role from the list. Next, participants were asked to individually resume the task, assuming the new role (or roles). We followed up with a rich open-ended inquiry of the participants' experiences around what happened when they stepped into a role, and concluded the interview with a discussion on creativity and the potential of assuming roles in general.

\section{Results}

Participants P1 (male, 19) and P2 (female, 23) were the ones who selected a set of multiple roles. Initially, P1 came up with eight ideas and P2 with six ideas. Having to choose from a list of roles while completing the task clearly did not help P1 and P2 much in the task, as P1 did not come up with any new ideas, whereas P2 also only managed to come up with one new idea. The two participants explained that they spent a considerable amount of effort on thinking what role would best fit the task at hand. The two participants had to ensure they could assume the role and know the role enough for it to be helpful in completing the task which clearly was not overall a good strategy. A further selection criteria for the role was that it had to be "open enough so that the brick could be re-purposed for the task" (P1). Both $\mathrm{P} 1$ and $\mathrm{P} 2$ reported that additional, and in this case harmful, considerations entered their thought process, resulting in being blocked or delayed in their creative thinking. P1 perhaps explained it best: 
"I clearly got stuck in having to choose a role, as I started to consider which of the roles I might have most experience or information about."

Participant P2 was also affected by choice overload [54]. She stated being over-committed to the role and thus "got blocked" on trying to relate the common object to a different role.

The other participants, however, concluded in unison that assuming a single role helped them significantly in coming up with new ideas. P3 (male, 21) and P4 (female, 23), for instance, managed to more than double the amount of their ideas by assuming a role after they had initially run out of new uses for the objects, from three initial ideas to nine total ideas, and from six to 12, respectively. Further, P3 noted how assuming a role "helped me to come up with surprising and weird ideas that actually relate to real-life use." P3 noted that the "scenarios" helped him "to drive it in the right direction" and in getting unstuck. P5 (male, 22) agreed that roles made it "easier to think about new ideas."

P8 (male, 29) noted that the role "contextualized and structured his thinking," forcing his line of thought to be more specific, compared to "coming up with ideas haphazardly and out of the blue" without the role. While the role helped P8 to come up with four more specific ideas over the five ideas that he already had, some of his ideas were similar in their meaning. For instance, he refined his idea of "supporting a desk" to "elevating a countertop for better ergonomics." Clearly, the roles helped P8 to come up with ideas that were more narrowly focused and specific to the role. P8, however, also noted that this narrow focus may be a disadvantage, if one is looking for broader and holistically applicable ideas. Based on our analysis, the ideas produced under the priming condition seemed to also be more articulate and detailed than the initial ideas. We acknowledge that validating this will require more studies, which we leave as future work.

Self-reflection and Development of Ideas. After noting the fact that there is nothing to stop people from doing such mental exercises on their own when working on tasks requiring creativity, the discussion turned into self-reflection on the human cognitive capacity and how little, in fact, we know on how to better exploit our brains in everyday settings. P4, for instance, was "surprised on how well [assuming a role] worked," while P3 stated that "I realized how the brain really seems equivalent to a search engine in that if you give it a specific task, it will come up with answers." P5 shared the sentiment, emphasising that creativity typically is fostered by a real problem-solving need: "if you have a problem you want to solve yourself, [roles] will stimulate you." Indeed, coming up with creative uses for a brick is not a real-world and relatable problem, per se, but when assuming the role of for instance a baker, one might well start to envision an entire host of problems where a brick could be useful.
When asked what could be improved to make roles useful for the task, the participants made a number of suggestions. First, the participants thought the extent to which assuming a role could stretch their divergent thinking abilities highly depends on the role. The main criteria for selecting the role was that it needs to relate to the task and the object. When asked about why assuming roles produced more ideas, P4 thought that being given "a more focused task makes it easier to come up with real, but unusual uses." $\mathrm{P} 4$ added that "broad topics" were not as helpful as specific ones and that a baker is about "general work" that she could not relate to. P2 also reported that he had trouble relating the chosen role to the task: "I thought the baker was unrelated to the paperclip and struggled to come up with more ideas." Second, P3 mentioned that expanding the roles with scenarios might be helpful for the given task: "Scenarios would help the participant in producing more ideas and in more detail." In other words, providing a specific context could be helpful. Furthermore, P3 mentioned that the paperclip can be seen as a tool, which he perceived as being helpful in coming up with uses for the object. Third, being given the role in the beginning would have been more helpful: "If I had been primed from the get go 'but only had a brick', I would have been more successful in embodying that role and coming up with more uses" (P1).

\section{DISCUSSION}

Given the inherent emphasis of crowdsourcing in collecting insights rapidly, inexpensively and accurately, it has been suggested as a key approach for creativity support [7, 71]. In our study we set to augment the individual's creative thinking process through adopting different roles.

The apparent undecidedness of participants was surprising. A part of the crowd workers in our online study seem to have been inspired by the role and the image, as suggested by the increase in variance in the time taken to complete the condition. The participants were engaged and concentrated. However, we could not significantly differentiate between the results provided in the different conditions in our study, even between assigning no role at all and having a role plus a stimulating image which, according to prior work, could have well led to a positive effect on creativity [40, 46, 63]. While the attitude towards the roles was slightly positive, the roles were not perceived as being strongly effective or even useful in accomplishing the task in the online study.

Methodologically, the interviews allowed us to both observe and inquire in an open-ended fashion about the effect of roles in a creative task, which ended up being highly insightful. For instance, noticing the participants having a clear revelation after assuming a role and continuing the task with new ideas would have been challenging to accomplish in an online labour platform. Yet, both the online study and interviews are crucial in understanding different aspects of 
the effects of roles on divergent thinking. While the arguably simple task in the online study did not manage to isolate an effect of roles, in the interviews we could clearly discover how they altered people's thinking and why.

While more studies are needed, our findings certainly suggest that roles can be useful but are not a silver bullet for augmenting an individual's creativity, especially in online crowdsourcing markets where people are typically hired to complete short tasks in a repeated fashion. In the Alternate Uses task, people go for "low-hanging fruit" first and deplete their repository of ideas that are easy to recall - a phenomenon called the path of least resistance [67]. On the other hand, the findings from the interviews are in line with related work on the crucial characteristics of context $[1,17]$ in crowdsourcing and with Amabile's framework for conceptualising creativity into domain-relevant skills, creativityrelevant skills, and task motivation as a set of necessary and sufficient components of creativity [4].

The participants in our second study who were given a list of roles reported having trouble making use of the roles as additional considerations entered their thought process, resulting in being blocked in producing ideas. Participants who were briefed about the task and then given the choice of selecting a single role, however, reported that the role helped them in coming up with more ideas, once their flow of ideas began to ebb away. Speculating further based on the interview results, setting limits might be helpful in fostering creativity. Too much liberty might backfire in form of participants getting stuck on making decisions rather than proceeding to complete the task at hand.

The results of our second study also highlight that, if given at the right time and for the right problem, roles constitute a tool for overcoming an impasse in the flow of ideas. Specific scenarios that prime participants to regard the role as a tool in a certain context may inspire the participants and produce answers with a more narrow focus. Further, having prior knowledge of the role seems to be advantageous: A familiar and "relatable" role helps more than a randomly assigned role in fostering one's divergent thinking process. What is remarkable and often neglected about this type of role-play is how it constitutes a free-of-cost and accessible tool by anyone in a variety of real-life situations. Indeed, assuming the role of others is nothing new to us humans, as that is the very mechanism that allows us to practice and learn empathy [15]. We just often fail to employ this tool in other areas of life, such as in creative pursuits.

\section{Creativity Studies on Crowdsourcing Platforms}

Creativity support tools, including crowdsourced solutions, enjoy increased traction in academia $[25,50,51]$. The experiences reported in this paper, however, lead us to critically examine the characteristics of crowdsourcing marketplaces for creativity studies. Researchers typically exploit one of the online labour markets, such as MTurk or Prolific. Research in the Social Sciences, in particular, has seen an increase in the use of MTurk and similar online platforms [6]. These online labour markets all suffer from various issues and challenges [14, 34]. For instance, the workers are a highly self-selected group of people (c.f. [16]) and researchers have to carefully monitor the work quality, e.g. with Instructional Manipulation Checks [49] and gold standard questions [18].

To this end, our findings suggest that conducting creativity research on these platforms is challenging for various other reasons. Crowd-powered creativity support tools operate in a space in which there is no right answer to a task, making the validation of work and the accuracy of workers far from trivial. Subjective tasks are prone to cheating [34]. In our studies, 16 workers, in fact, confessed to searching for answers online. Further, crowdsourcing platforms largely consist of a nonreplenishing subject pool [14] making participants' nonnaïveté a challenge for studies involving creativity in these platforms. Eleven participants in our study mentioned they had completed the Alternate Uses test with the same object before. Clearly, these biases are difficult to prevent, but at the same time preventing such bias is a precondition for creativity-related studies to produce valid results.

Yet, the convenience and accessibility of crowdsourcing platforms make them compelling options for running studies similar to ours, or building creativity support systems in which repetitive creative tasks are completed under different mental or digital contexts. We provide design recommendations for these studies and systems in the next section.

\section{Design Recommendations for Using Roles in Computational Priming}

The contrast between our two studies revealed important considerations for the use of computational priming and the design of creativity support tools. Based on the findings and lessons-learned from our studies, we provide recommendations for the use of roles in creativity studies on crowdsourcing platforms and in crowd-powered creativity support tools.

Choose the right time point for priming. Priming may be a way to combat a decline in productivity [40]. A key element therefore is choosing the right time point for computational priming. Supported by prior work [65], our findings indicate that workers may be most conducive to being primed when they reach an impasse in the flow of ideas. While we found evidence that priming workers from the very start may more narrowly scope the answers provided, the task outcome may not be more creative. This finding implicates that creativity support tools should monitor the productivity of their users to identify the right time point for computational priming to overcome an impasse in the flow of ideas. 
Let workers self-select a suitable role. Timely on-demand access to stimuli has been proven to be beneficial in brainstorming [58]. In our study, participants who selected a single role performed better than participants who selected multiple roles. There are, however, other factors to consider when selecting roles. The framing of the task should feed into the person's expertise and skills in creative thinking [5]. The wrong role may lead to an increase in cognitive stress due to thinking that the ideas must be related to this role or simply not having enough information about the role. Further, priming cannot help with impossible tasks, i.e., tasks in which the person would first need to acquire new knowledge [46]. Being given the wrong role may therefore reduce a person's capacity to come up with ideas. Supported by the suggestion in related literature to give people autonomy concerning the means, but not necessarily the ends [5], one way of assuring that a person knows the role is to let the person self-select the role. We believe the strategy of self-selecting a single role may be more effective than providing a detailed description of the role because this strategy ensures a good fit between the role and the person selecting the role. Another approach would be to automatically assign a role based on the worker's profile, e.g. from information extracted from social networks [24].

A narrowly focused task may inspire more targeted ideas. A more focused task may make it easier for workers to come up with real, but unusual ideas. Further, we speculate that framing a task with a sense of urgency may lead workers to produce more creative ideas at a higher level of detail. Task instructions, such as "assume you are a construction worker and you only have a brick" or "imagine you are stranded on an island and the only object you have available is a paperclip" may be more effective than less detailed task instructions.

Prevent over-commitment to the role. Designers of creativity support tools should aim to prevent workers from overcommitting to the role, which may lead workers to get stuck or blocked in their train of thought. Workers should be allowed to deviate from a given role, if they believe it advances their creative output. Further, designers should consider the ethical implications of computational priming. For a short discussion of ethical implications of computational priming see [46].

Prevent over-exposure to other contexts. Last, online workers may be affected by a multitude of stimuli due to multitasking [40]. Workers on crowdsourcing platforms, in particular, are known to engage in multitasking as a strategy to maximise their earnings [30, 38]. It is possible that multitasking may reduce the effectiveness of computational priming on crowdsourcing platforms because the context of workers cannot be controlled. Measures could be implemented to monitor the multitasking behaviour of workers, for instance to detect if the worker potentially becomes exposed to other stimuli and contexts.

\section{Limitations}

We acknowledge certain key limitations in our study. First, workers were not specifically instructed to associate their ideas with the given role. While this decision did not restrict the free will, it may have confused some of the participants. Further, the examples given in the task instructions may have had an anchoring effect on some participants.

Second, prior research has shown the order of microtasks may impact the work quality [10, 47, 63]. To minimise intertask effects in our study, we counterbalanced the order of the conditions in the study design. Nevertheless, it is likely that some participants just continued with their train of thought in subsequent stages and may thus have not been affected by the role as much as we expected.

Last, abrupt contextual switches may be counterproductive $[44,69]$. For instance, contextual switches may confuse workers and reduce their performance [39]. Inspiration from far domains may also harm creativity [12]. We chose to conduct a within-subject study in hope of more clearly demonstrating the effect of roles to the workers. While this did not take place at a magnitude we expected, we believe that the context switch itself did not play a role in the success, or lack thereof, of our study.

\section{CONCLUSION}

We investigated the effect of impersonating a role on the creativity of an individual in two complementary studies. In the first study, we invited 60 workers from an online labour market to complete a simple divergent thinking task. Each online worker completed the task without any assigned role, with a role, and with a role and an additional stimulus (in the form of an image depicting the role). In our second study, we investigated the use of roles in one's perception of the divergent thinking process.

Contrary to related work, our results indicate that in an online setting while working on a small batch of simple creative tasks, neither roles nor an additional priming image had a significant effect on the creativity of the ideas generated by the crowd workers. Our qualitative inquiry in the interviews, on the other hand, suggests that roles facilitate one's divergent thinking. Our results may inform the design of creativity studies in crowdsourcing marketplaces and the design of crowd-powered creativity support tools.

\section{ACKNOWLEDGMENTS}

This work is partly funded by the Academy of Finland (Grants 313224-STOP, 320089-SENSATE, 316253-SENSATE and 318927-6Genesis Flagship). 


\section{REFERENCES}

[1] Gregory D. Abowd, Anind K. Dey, Peter J. Brown, Nigel Davies, Mark Smith, and Pete Steggles. 1999. Towards a Better Understanding of Context and Context-Awareness. In Proceedings of the 1st International Symposium on Handheld and Ubiquitous Computing (HUC '99). Springer Verlag, London, UK, 304-307. https://doi.org/10.1007/3-540-481575_29

[2] Icek Ajzen. 1991. The Theory of Planned Behavior. Organizational Behavior and Human Decision Processes 50, 2 (1991), 179-211. https: //doi.org/10.1016/0749-5978(91)90020-T

[3] Niek Althuizen and Astrid Reichel. 2016. The Effects of IT-Enabled Cognitive Stimulation Tools on Creative Problem Solving: A Dual Pathway to Creativity. Journal of Management Information Systems 33, 1 (2016), 11-44. https://doi.org/10.1080/07421222.2016.1172439

[4] Teresa M. Amabile. 1983. The Social Psychology of Creativity: A Componential Conceptualization. Fournal of Personality and Social Psychology 45, 2 (1983), 357-377. https://doi.org/10.1037/0022-3514. 45.2.357

[5] Teresa M. Amabile. 1998. How to Kill Creativity. Harvard Business Review 76, 5 (1998), 76-87.

[6] Craig A. Anderson, Johnie J. Allen, Courtney Plante, Adele QuigleyMcBride, Alison Lovett, and Jeffrey N. Rokkum. 2019. The MTurkification of Social and Personality Psychology. Personality and Social Psychology Bulletin 45, 6 (2019), 842-850. https://doi.org/10.1177/ 0146167218798821

[7] Salvatore Andolina, Hendrik Schneider, Joel Chan, Khalil Klouche, Giulio Jacucci, and Steven Dow. 2017. Crowdboard: Augmenting InPerson Idea Generation with Real-Time Crowds. In Proceedings of the 2017 Conference on Creativity and Cognition (C\&C '17). ACM, New York, NY, USA, 106-118. https://doi.org/10.1145/3059454.3059477

[8] Tal August, Nigini Oliveira, Chenhao Tan, Noah Smith, and Katharina Reinecke. 2018. Framing Effects: Choice of Slogans Used to Advertise Online Experiments Can Boost Recruitment and Lead to Sample Biases. Proc. ACM Hum.-Comput. Interact. 2, CSCW (2018), 22:1-22:19. https: //doi.org/10.1145/3274291

[9] Tal August and Katharina Reinecke. 2019. Pay Attention, Please: Formal Language Improves Attention in Volunteer and Paid Online Experiments. In Proceedings of the 2019 CHI Conference on Human Factors in Computing Systems (CHI '19). ACM, New York, NY, USA, 248:1-248:11. https://doi.org/10.1145/3290605.3300478

[10] Carrie J. Cai, Shamsi T. Iqbal, and Jaime Teevan. 2016. Chain Reactions: The Impact of Order on Microtask Chains. In Proceedings of the 2016 CHI Conference on Human Factors in Computing Systems (CHI '16). ACM, New York, NY, USA, 3143-3154. https://doi.org/10.1145/2858036. 2858237

[11] Erin A. Carroll, Celine Latulipe, Richard Fung, and Michael Terry. 2009. Creativity Factor Evaluation: Towards a Standardized Survey Metric for Creativity Support. In Proceedings of the 7th Conference on Creativity and Cognition (C\&C '09). ACM, New York, NY, USA, 127-136. https://doi.org/10.1145/1640233.1640255

[12] Joel Chan, Pao Siangliulue, Denisa Qori McDonald, Ruixue Liu, Reza Moradinezhad, Safa Aman, Erin T. Solovey, Krzysztof Z. Gajos, and Steven P. Dow. 2017. Semantically Far Inspirations Considered Harmful?: Accounting for Cognitive States in Collaborative Ideation. In Proceedings of the 2017 Conference on Creativity and Cognition (C\&C '17) ACM, New York, NY, USA, 93-105. https://doi.org/10.1145/3059454. 3059455

[13] Dana Chandler and Adam Kapelner. 2013. Breaking Monotony with Meaning: Motivation in Crowdsourcing Markets. Fournal of Economic Behavior \& Organization 90 (2013), 123-133. https://doi.org/10.1016/j. jebo.2013.03.003
[14] Jesse Chandler, Pam Mueller, and Gabriele Paolacci. 2014. Nonnaïveté among Amazon Mechanical Turk Workers: Consequences and Solutions for Behavioral Researchers. Behavior Research Methods 46, 1 (2014), 112-130. https://doi.org/10.3758/s13428-013-0365-7

[15] Gerald L. Clore and Katharine M. Jeffery. 1972. Emotional Role Playing, Attitude Change, and Attraction Toward a Disabled Person. Journal of Personality and Social Psychology 23, 1 (1972), 105-111. https: //doi.org/10.1037/h0032867

[16] Mick P. Couper. 2000. Web Surveys: A Review of Issues and Approaches. Public Opinion Quarterly 64, 4 (2000), 464-494. https: //doi.org/10.1086/318641

[17] Felipe A. Csaszar and J. P. Eggers. 2013. Organizational Decision Making: An Information Aggregation View. Management Science 59, 10 (2013), 2257-2277. https://doi.org/10.1287/mnsc.1120.1698

[18] Florian Daniel, Pavel Kucherbaev, Cinzia Cappiello, Boualem Benatallah, and Mohammad Allahbakhsh. 2018. Quality Control in Crowdsourcing: A Survey of Quality Attributes, Assessment Techniques, and Assurance Actions. ACM Comput. Surv. 51, 1 (2018), 7:1-7:40. https://doi.org/10.1145/3148148

[19] Fred D. Davis. 1989. Perceived Usefulness, Perceived Ease of Use, and User Acceptance of Information Technology. MIS Quarterly 13, 3 (1989), 319-340. https://doi.org/10.2307/249008

[20] Alwin de Rooij, Sarah van der Land, and Shelly van Erp. 2017. The Creative Proteus Effect: How Self-Similarity, Embodiment, and Priming of Creative Stereotypes with Avatars Influences Creative Ideation. In Proceedings of the 2017 Conference on Creativity and Cognition (C\&C '17). ACM, New York, NY, USA, 232-236. https://doi.org/10.1145/ 3059454.3078856

[21] Alan R. Dennis, Randall K. Minas, and Akshay P. Bhagwatwar. 2013. Sparking Creativity: Improving Electronic Brainstorming with Individual Cognitive Priming. Journal of Management Information Systems 29, 4 (2013), 195-216. https://doi.org/10.2753/MIS0742-1222290407

[22] Alan R. Dennis and Mike L. Williams. 2003. Electronic Brainstorming: Theory, Research, and Future Directions. In Group Creativity: Innovation through Collaboration, Paul B. Paulus and Bernard A. Nijstad (Eds.). Oxford University Press, New York, NY, USA, 160-178. https://doi.org/10.1093/acprof:oso/9780195147308.003.0008

[23] Anind K. Dey. 2001. Understanding and Using Context. Personal Ubiquitous Comput. 5, 1 (2001), 4-7. https://doi.org/10.1007/s007790170019

[24] Djellel Eddine Difallah, Gianluca Demartini, and Philippe CudréMauroux. 2013. Pick-a-crowd: Tell Me What You Like, and I'll Tell You What to Do. In Proceedings of the 22nd International Conference on World Wide Web (WWW'13). ACM, New York, NY, USA, 367-374. https://doi.org/10.1145/2488388.2488421

[25] Jonas Frich, Lindsay MacDonald Vermeulen, Christian Remy, Michael Mose Biskjaer, and Peter Dalsgaard. 2019. Mapping the Landscape of Creativity Support Tools in HCI. In Proceedings of the 2019 CHI Conference on Human Factors in Computing Systems (CHI '19). ACM, New York, NY, USA, 389:1-389:18. https://doi.org/10.1145/3290605. 3300619

[26] Ujwal Gadiraju, Ricardo Kawase, and Stefan Dietze. 2014. A Taxonomy of Microtasks on the Web. In Proceedings of the 25th ACM Conference on Hypertext and Social Media (HT '14). ACM, New York, NY, USA, 218-223. https://doi.org/10.1145/2631775.2631819

[27] Ujwal Gadiraju, Sebastian Möller, Martin Nöllenburg, Dietmar Saupe, Sebastian Egger-Lampl, Daniel Archambault, and Brian Fisher. 2017. Crowdsourcing Versus the Laboratory: Towards Human-Centered Experiments Using the Crowd. In Evaluation in the Crowd. Crowdsourcing and Human-Centered Experiments, Daniel Archambault, Helen Purchase, and Tobias Hoßfeld (Eds.). Springer International Publishing, Cham, 6-26. https://doi.org/10.1007/978-3-319-66435-4_2

[28] Joy Paul Guilford, Paul R. Christensen, Philip R. Merrifield, and Robert C. Wilson. 1978. Alternate Uses: Manual of Instructions and 
Interpretation. Sheridan Psychological Services, Orange, CA, USA.

[29] Juho Hamari, David J. Shernoff, Elizabeth Rowe, Brianno Coller, Jodi Asbell-Clarke, and Teon Edwards. 2016. Challenging Games Help Students Learn: An Empirical Study on Engagement, Flow and Immersion in Game-based Learning. Computers in Human Behavior 54 (2016), 170-179. https://doi.org/10.1016/j.chb.2015.07.045

[30] Kotaro Hara, Abigail Adams, Kristy Milland, Saiph Savage, Chris Callison-Burch, and Jeffrey P. Bigham. 2018. A Data-Driven Analysis of Workers' Earnings on Amazon Mechanical Turk. In Proceedings of the 2018 CHI Conference on Human Factors in Computing Systems (CHI '18). ACM, New York, NY, USA, 449:1-449:14. https: //doi.org/10.1145/3173574.3174023

[31] Stefan M. Herzog and Ralph Hertwig. 2009. The Wisdom of Many in One Mind: Improving Individual Judgments With Dialectical Bootstrapping. Psychological Science 20, 2 (2009), 231-237. https://doi.org/ 10.1111/j.1467-9280.2009.02271.x

[32] Tom Hewett, Mary Czerwinski, Michael Terry, Jay Nunamaker, Linda Candy, Bill Kules, and Elisabeth Sylvan. 2005. Creativity Support Tool Evaluation Methods and Metrics. In Report From a U.S. National Science Foundation Sponsored Workshop on Creativity Support Tools, Ben Shneiderman, Gerhard Fischer, Mary Czerwinski, Brad Myers, and Mitch Resnick (Eds.). University of Maryland, 10-24.

[33] Jeff Howe. 2006. The Rise of Crowdsourcing. Wired Magazine 14, 6 (2006), 1-4.

[34] Aniket Kittur. 2010. Crowdsourcing, Collaboration and Creativity. XRDS 17, 2 (2010), 22-26. https://doi.org/10.1145/1869086.1869096

[35] Aniket Kittur, Ed H. Chi, and Bongwon Suh. 2008. Crowdsourcing User Studies with Mechanical Turk. In Proceedings of the SIGCHI Conference on Human Factors in Computing Systems (CHI '08). ACM, New York, NY, USA, 453-456. https://doi.org/10.1145/1357054.1357127

[36] Richard P. Larrick and Jack B. Soll. 2006. Intuitions About Combining Opinions: Misappreciation of the Averaging Principle. Management Science 52, 1 (2006), 111-127. https://doi.org/10.1287/mnsc.1050.0459

[37] Richard P. Larrick and Jack B. Soll. 2011. The Social Psychology of the Wisdom of Crowds. In Social fudgment and Decision Making, Joachim I. Krueger (Ed.). Psychology Press, New York, NY, 227-242. https://doi.org/10.4324/9780203854150

[38] Laura Lascau, Sandy J. J. Gould, Anna L. Cox, Elizaveta Karmannaya, and Duncan P. Brumby. 2019. Monotasking or Multitasking: Designing for Crowdworkers' Preferences. In Proceedings of the ACM CHI Conference on Human Factors in Computing Systems (CHI '19). ACM, New York, NY, USA. https://doi.org/10.1145/3290605.3300649

[39] Walter S. Lasecki, Jeffrey M. Rzeszotarski, Adam Marcus, and Jeffrey P. Bigham. 2015. The Effects of Sequence and Delay on Crowd Work. In Proceedings of the 33rd Annual ACM Conference on Human Factors in Computing Systems (CHI '15). ACM, New York, NY, USA, 1375-1378. https://doi.org/10.1145/2702123.2702594

[40] Sheena Lewis, Mira Dontcheva, and Elizabeth M. Gerber. 2011. Affective Computational Priming and Creativity. In Proceedings of the SIGCHI Conference on Human Factors in Computing Systems (CHI '11). ACM, New York, NY, USA, 735-744. https://doi.org/10.1145/1978942. 1979048

[41] Rhema Linder, Clair Snodgrass, and Andruid Kerne. 2014. Everyday Ideation: All of My Ideas Are on Pinterest. In Proceedings of the 32nd Annual ACM Conference on Human Factors in Computing Systems (CHI '14). ACM, New York, NY, USA, 2411-2420. https://doi.org/10.1145/ 2556288.2557273

[42] Albert E. Mannes, Jack B. Soll, and Richard P. Larrick. 2014. The Wisdom of Select Crowds. Fournal of Personality and Social Psychology 107, 2 (2014), 276-299. https://doi.org/10.1037/a0036677

[43] Manon Marinussen and Alwin de Rooij. 2019. Being Yourself to Be Creative: How Self-Similar Avatars Can Support the Generation of
Original Ideas in Virtual Environments. In Proceedings of the 2019 on Creativity and Cognition (C\&C '19). ACM, New York, NY, USA, 285-293. https://doi.org/10.1145/3325480.3325482

[44] Gloria Mark, Daniela Gudith, and Ulrich Klocke. 2008. The Cost of Interrupted Work: More Speed and Stress. In Proceedings of the SIGCHI Conference on Human Factors in Computing Systems (CHI '08). ACM, New York, NY, USA, 107-110. https://doi.org/10.1145/1357054.1357072

[45] David L. Morgan, Jutta Ataie, Paula Carder, and Kim Hoffman. 2013. Introducing Dyadic Interviews as a Method for Collecting Qualitative Data. Qualitative Health Research 23, 9 (2013), 1276-1284. https: //doi.org/10.1177/1049732313501889

[46] Robert R. Morris, Mira Dontcheva, and Elizabeth M. Gerber. 2012. Priming for Better Performance in Microtask Crowdsourcing Environments. IEEE Internet Computing 16, 5 (2012), 13-19. https: //doi.org/10.1109/MIC.2012.68

[47] Edward Newell and Derek Ruths. 2016. How One Microtask Affects Another. In Proceedings of the 2016 CHI Conference on Human Factors in Computing Systems (CHI '16). ACM, New York, NY, USA, 3155-3166. https://doi.org/10.1145/2858036.2858490

[48] Thi Thao Duyen T. Nguyen, Thomas Garncarz, Felicia Ng, Laura A. Dabbish, and Steven P. Dow. 2017. Fruitful Feedback: Positive Affective Language and Source Anonymity Improve Critique Reception and Work Outcomes. In Proceedings of the 2017 ACM Conference on Computer Supported Cooperative Work and Social Computing (CSCW '17). ACM, New York, NY, USA, 1024-1034. https://doi.org/10.1145/ 2998181.2998319

[49] Daniel M. Oppenheimer, Tom Meyvis, and Nicolas Davidenko. 2009. Instructional Manipulation Checks: Detecting Satisficing to Increase Statistical Power. Fournal of Experimental Social Psychology 45, 4 (2009), 867-872. https://doi.org/10.1016/j.jesp.2009.03.009

[50] Jonas Oppenlaender, Maximilian Mackeprang, Abderrahmane Khiat, Maja Vuković, Jorge Goncalves, and Simo Hosio. 2019. DC2S2: Designing Crowd-powered Creativity Support Systems. In Extended Abstracts of the 2019 CHI Conference on Human Factors in Computing Systems (CHI EA '19). ACM, New York, NY, USA, W06:1-W06:8. https://doi.org/10.1145/3290607.3299027

[51] Jonas Oppenlaender, Naghmi Shireen, Maximilian Mackeprang, Halil Erhan, Jorge Goncalves, and Simo Hosio. 2019. Crowd-powered Interfaces for Creative Design Thinking. In Proceedings of the 2019 Conference on Creativity and Cognition (C\&C '19). ACM, New York, NY, USA, 722-729. https://doi.org/10.1145/3325480.3326553

[52] Eyal Peer, Laura Brandimarte, Sonam Samat, and Alessandro Acquisti. 2017. Beyond the Turk: Alternative Platforms for Crowdsourcing Behavioral Research. Journal of Experimental Social Psychology 70 (2017), 153-163. https://doi.org/10.1016/j.jesp.2017.01.006

[53] Dražen Prelec, H. Sebastian Seung, and John McCoy. 2017. A Solution to the Single-question Crowd Wisdom Problem. Nature 541 (2017), 532-535. https://doi.org/10.1038/nature21054

[54] Barry Schwartz. 2004. The Paradox of Choice: Why More Is Less. Ecco, New York, NY, USA.

[55] David J. Shernoff. 2013. Optimal Learning Environments to Promote Student Engagement. Springer, New York, NY, USA.

[56] Ben Shneiderman. 2002. Creativity Support Tools. Commun. ACM 45, 10 (2002), 116-120. https://doi.org/10.1145/570907.570945

[57] Ben Shneiderman. 2007. Creativity Support Tools: Accelerating Discovery and Innovation. Commun. ACM 50, 12 (2007), 20-32. https://doi.org/10.1145/1323688.1323689

[58] Pao Siangliulue, Joel Chan, Krzysztof Z. Gajos, and Steven P. Dow. 2015. Providing Timely Examples Improves the Quantity and Quality of Generated Ideas. In Proceedings of the 2015 ACM SIGCHI Conference on Creativity and Cognition (C\&C '15). ACM, New York, NY, USA, 83-92. https://doi.org/10.1145/2757226.2757230 
[59] Paul J. Silvia, Beate P. Winterstein, John T. Willse, Christopher M. Barona, Joshua T. Cram, Karl I. Hess, Jenna L. Martinez, and Crystal A. Richard. 2008. Assessing Creativity with Divergent Thinking Tasks: Exploring the Reliability and Validity of New Subjective Scoring Methods. Psychology of Aesthetics, Creativity, and the Arts 2, 2 (2008), 68-85. https://doi.org/10.1037/1931-3896.2.2.68

[60] Paul J. Silvia, Beate P. Winterstein, John T. Willse, Christopher M. Barona, Joshua T. Cram, Karl I. Hess, Jenna L. Martinez, and Crystal A. Richard. 2008. Assessing Creativity with Divergent Thinking Tasks: Exploring the Reliability and Validity of New Subjective Scoring Methods. Psychology of Aesthetics, Creativity, and the Arts 2, 2 (2008), 68-85.

[61] Allan Snyder, John Mitchell, Terry Bossomaier, and Gerry Pallier. 2004 The Creativity Quotient: An Objective Scoring of Ideational Fluency. Creativity Research fournal 16, 4 (2004), 415-419. https://doi.org/10. 1080/10400410409534552

[62] James Surowiecki. 2005. The Wisdom of Crowds. Anchor, New York, NY, USA.

[63] Jaime Teevan and Lisa Yu. 2017. Bringing the Wisdom of the Crowd to an Individual by Having the Individual Assume Different Roles. In Proceedings of the 2017 Conference on Creativity and Cognition (C\&C '17). ACM, New York, NY, USA, 131-135. https://doi.org/10.1145/ 3059454.3059467

[64] Stefan Thomke and Eric von Hippel. 2002. Customers as Innovators: A New Way to Create Value. Harvard Business Review 80, 4 (2002), $51-61$.
[65] Edward Vul and Harold Pashler. 2008. Measuring the Crowd within: Probabilistic Representations within Individuals. Psychological Science 19, 7 (2008), 645-647. https://doi.org/10.1111/j.1467-9280.2008.02136.x

[66] Kai Wang and Jeffrey V. Nickerson. 2017. A Literature Review on Individual Creativity Support Systems. Computers in Human Behavior 74 (2017), 139-151. https://doi.org/10.1016/j.chb.2017.04.035

[67] Thomas B. Ward. 1994. Structured Imagination: The Role of Category Structure in Exemplar Generation. Cognitive Psychology 27, 1 (1994), 1-40. https://doi.org/10.1006/ cogp.1994.1010

[68] Robert W. Weisberg. 2006. Creativity: Understanding Innovation in Problem Solving, Science, Invention and the Arts. John Wiley \& Sons, Hoboken, NJ, USA.

[69] Glenn Wylie and Alan Allport. 2000. Task Switching and the Measurement of "Switch Costs". Psychological Research 63, 3 (2000), 212-233. https://doi.org/10.1007/s004269900003

[70] Lixiu Yu, Aniket Kittur, and Robert E. Kraut. 2014. Searching for Analogical Ideas with Crowds. In Proceedings of the 32nd Annual ACM Conference on Human Factors in Computing Systems (CHI '14). ACM, New York, NY, USA, 1225-1234. https://doi.org/10.1145/2556288.2557378

[71] Lixiu Yu, Aniket Kittur, and Robert E. Kraut. 2016. Encouraging "Outside-The-Box" Thinking in Crowd Innovation Through Identifying Domains of Expertise. In Proceedings of the 19th ACM Conference on Computer-Supported Cooperative Work \& Social Computing (CSCW '16). ACM, New York, NY, USA, 1214-1222. https://doi.org/10.1145/ 2818048.2820025 